
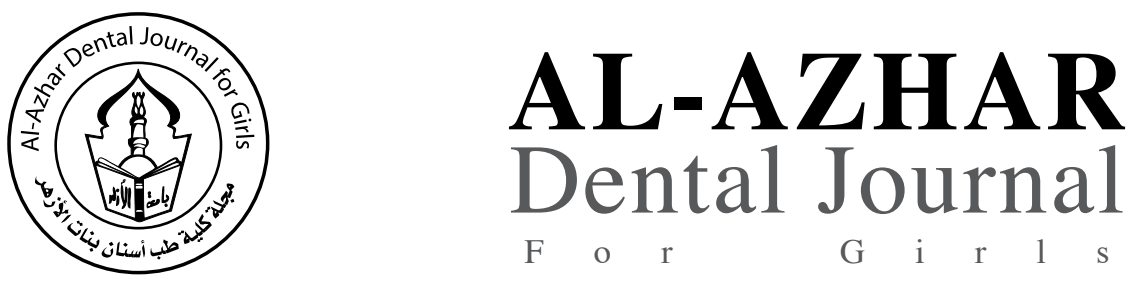

The Official Publication of The Faculty of Dental Medicine For Girls, Al-Azhar University Cairo, Egypt.

ADJ-for Grils, Vol. 4, No. 2, April (2017) — PP. 121:126

\title{
Clinical Evaluation of Single versus Multiple Visits Endodontic Retreatment Outcome: A Randomized Controlled Trial
}

\author{
Hemat M. EL Sheikh ${ }^{(1)}$, Mervat I. Fawzy ${ }^{(2)}$, Mohamed M. Khalefa ${ }^{(2)}$ and Hagar A. Bastawy ${ }^{(3)}$
}

Codex : 14/1704

dentaljournal.forgirls@yahoo.com

\begin{abstract}
Aim: This study was conducted to evaluate the post-operative pain following single versus multiple visits endodontic retreatment. Materials and Method: Fifty cases of single rooted teeth in (29) patients had failed endodontic treatment were selected according to inclusion criteria. Using simple randomization by table, the selected patients were divided into two groups, where Group I: the retreatment was performed in single visit. Group II; the retreatment was performed in two visits and $\mathrm{Ca}(\mathrm{OH})_{2}$ mixed with $2 \%$ Chlorhxidin was used as intracanal medicament. The outcome of the retreatment was evaluated by recording post-operative pain and flare up. Results: There was no statistical significant difference in post-operative pain intensity between single and multiple visits endodontic retreatment after 24 hours as well as 7 days $(\mathrm{P} \leq 0.05)$.The pain recorded was in mild category.
\end{abstract}

\section{INTRODUCTION}

Contemporary endodontic treatment aims to preserve functionality and aesthetics of teeth with diseased pulp and/ or periapical tissues. Endodontic treatment is a conservative modality yielding a high long term tooth survival ${ }^{(1)}$. The outcome of endodontic treatment performed in controlled clinical environment have demonstrated success rate around $90 \%$.In certain cases endodontic treatment may fail, one of the most common reasons is inability to eliminate microorganisms from the root canal. When non-surgical root canal therapy fails, the treatment options include orthograd re-treatment, periradicular surgery, or extraction $^{(2-3)}$.

1. Assistant Lecturer of Endodontics, Faculty of Dental Medicine (for Girls), AL-Azhar University.

2. Professor of Endodontics, Faculty of Dental Medicine (for Girls), Al Azhar University.

3. Lecturer of Endodontics, Faculty of Dental Medicine (for Girls), Al Azhar University. 
Even with the outmost care in performing a root canal treatment, some patients experience pain after treatment. Post-operative pain is an unpleasant situation for both the dentist and patient; it affects patient's quality of life and the success of dentist patient relationship. The pain intensity can range from mild to severe, the occurrence of mild pain is relatively common even when the treatment has followed the highest standards and should be expected and anticipated by patients. According to previous published data, frequency of post endodontic pain ranges from 1.4 to $16 \%$ and sometimes up to $50 \%$ in some studies ${ }^{(4-7)}$.

Despite carful instrumentation and irrigation of root canal system, antimicrobial agents have been used for further disinfection to enhance the chance for treatment success. Calcium hydroxide mixed with $2 \%$ chlorhexidine has proven to be an excellent antimicrobial agent for intracanal dressing, which contributes to the maintenance of bacteria-free root canal after completed endodontic therapy. Thus, multiple-visits root canal treatment is accepted as a safe and common therapy. Furthermore, when flareups occur during multiple-visit procedures, they can be addressed prior to obturation ${ }^{(8-10)}$.

A growing perception in endodontic is that root canal therapy can be performed in one endodontic visit.it reduces the number of patient appointments, eliminates the chance for inter-appointment microbial contamination, it allows the endodontists perform the root canal filling when they are more familiar with the canal anatomy, and reduces procedural costs ${ }^{(11)}$.

Some authors studied the post-obturation pain after single and multiple endodontic treatment, they concluded that there was no difference in the postoperative pain between patients treated in one-visit and patients treated in two appointments. The majority of patients in both groups reported no pain or only minimal pain within 24 to 48 hours of treatment ${ }^{(12-14)}$.While some researchers presented a higher incidence of post-obturation pain following single visit root canal treatment, they reported higher incidences for post-obturation pain and flare-ups following the single visit procedure ${ }^{(15)}$.Other studies presented higher incidence of post-obturation pain following two visits root canal treatment ${ }^{(16-17)}$.

One study evaluated post-obturation pain after single versus multiple endodontic retreatment, they reported that two-visit endodontic treatment with intracanal medication was found to be effective in reducing postoperative pain of previously symptomatic teeth and decreased the number of flareups in all retreatment cases ${ }^{(18)}$. Thus, the aim of the present randomized clinical trial was to evaluate the incidence and the level of post-operative pain in endodontic retreatment after single versus two visits technique.

\section{MATERIALS AND METHODS}

Fifty cases of single rooted teeth that had failed endodontic treatment from 29 patients were enrolled in this study which was approved by the research ethics committee of Faculty of Dental Medicine for Girls Al-Azhar University. The patients assessed for eligibility were informed on the objective and design of the study, and gave their consent for the retreatment by signing a dedicated form.

\section{Inclusion criteria:}

- Patients had anon contributory medical history.

- Patients of age categories between 15 to 40 years old.

- Cases of failed endodontic treatment resulting from inadequate root canal filling.

- $\quad$ Single rooted teeth with root canal form type I.

\section{Exclusion criteria:}

The cases representing clinical complains as following:

- Tenderness to percussion and palpation of adjacent soft tissue. 
- Presence of an associated sinus tract.

- Presence of swelling in adjacent soft tissue.

- Teeth with periodontal pathologies or mobility.

- Teeth representing root fracture.

- Pregnant women.

\section{Diagnosis of cases:}

A complete history was obtained and clinical examination was conducted on each of the selected case. Retreatment decision was based on detailed analysis of clinical examination, intraoral radiograph.

\section{Treatment protocol:}

The fifty teeth (23maxillay anteriors, 13 mandibular anteriors, 4 maxillary premolars and 10 mandibular premolars) were divided randomly into two groups (25 teeth each).Method of simple table randomization was used for assigning the patients randomly to the two study groups depending on number of treatment visits.

Group I: Teeth were treated in single visit. Group II: Teeth were treated in multiple (two) visits

The clinical question in this study was addressed in terms of PICO question which involves 4 elements: [problem $(\mathrm{P})$, intervention (I), comparison $(\mathrm{C})$ and outcome $(\mathrm{O})]$ as following:

P. Failed endodontically treated teeth (problem).

I. Root canal retreatment (intervention).

C. Single versus multiple retreatment sessions (comparison).

O. Post-operative pain and flare up (outcome).

\section{Group I:}

The retreatment procedures including coronal access reopening and adjusting, removal of old root canal filling materials using Gates- Glidden, Hedstroem files and d-limonene (Carvene) GP solvent. Working length determination using apex locator and periapical radiograph. Preparation of root canal system using hybrid technique, and the irrigation was performed using $2.5 \% \mathrm{NaOCl}$ and $17 \%$ EDTA solution. The obturation was performed with gutta-percha and AH Plus sealer using lateral condensation technique. The direct final restoration was performed at the same visit and indirect restoration was performed one week after.

\section{Group II:}

All treatment procedures performed in group I was followed in group II but after mechanical preparation and irrigation of root canal system $\mathrm{Ca}$ $(\mathrm{OH})_{2}$ was mixed with $2 \% \mathrm{CHX}$ liquid and applied to root canal using master apical file. In next visit one week after $\mathrm{Ca}(\mathrm{OH})_{2}$ was removed using $\mathrm{K}$ files and $2.5 \% \mathrm{NaOCl}$. The obturation and coronal restoration was then performed as the same as in group I.

\section{Follow up and outcome evaluation}

Patients were recalled after obturation and final restoration for groups (I\&II), after $24 \mathrm{hrs,} \mathrm{one} \mathrm{week,}$ and one month. At each recall appointment they were instructed to fill a self-report questionnaire for assessment of post-operative pain using visual analogue scale (VAS) ${ }^{(19)}$.

\section{Statistical analysis}

Numerical data were explored for normality by checking the data distribution and using Kolmogorov-Smirnov and Shapiro-Wilk tests.

\section{RESULTS}

There was no statistically significant difference in pain intensity between the single and multiple visits groups after 24 hours as well as one week ( $\mathrm{P}$ $\leq 0.05$ ). However, after 24 hours; number of patients with no pain in group I were equal to that in group II, while number of patients with mild pain were lower than patients in Group II. After one week, all 
patients in group I showed no pain and in group II only one patient with mild pain. After 1 month, all patients in groups I\&II had no pain.

Within each group, there was no statistically significant increase in number of patients with no pain in single visit group (from17 to 20) from 24 hours to one week ( $\mathrm{P} \leq 0.05)$. However, there was statistically significant increase in number of patients with no pain in multiple-visits group (from 17 to 22$)$ from 24 hours to one week $(\mathrm{P} \leq 0.05)$.Pain disappeared from 24 hours to one week in group I $100 \%$ however, in group II $95.6 \%$ with no pain.

There was no patient reported in moderate or severe pain categories in single visit and I multiple visits groups through whole periods of study intervals.

Table (1) Frequencies (n), percentages (\%) and P-value for the changes in pain intensity within each Group (1,II)

\begin{tabular}{c|c|c|c|c|c|c|c}
\hline & \multicolumn{2}{|c|}{24 Hours } & \multicolumn{2}{c|}{1 Week } & \multicolumn{2}{c|}{1 Month } & \multirow{2}{*}{ P-value } \\
\cline { 2 - 7 } & $\mathrm{n}$ & $\%$ & $\mathrm{n}$ & $\%$ & $\mathrm{n}$ & $\%$ & \\
\hline Group I & & & & & & \\
No pain & 17 & 85.0 & 20 & 100.0 & 20 & 100.0 & $\mathbf{0 . 0 8 3}$ \\
Mild pain & 3 & 15.0 & 0 & 0.0 & 0 & 0.0 & \\
Group II & & & & & & \\
No pain & 17 & 73.9 & 22 & 95.6 & 23 & 100.0 & \multirow{2}{*}{$\mathbf{0 . 0 2 5}$} \\
Mild pain & 6 & 26.1 & 1 & 4.4 & 0 & 0.0 & \\
\hline
\end{tabular}

*: Significant at $P \leq 0.05$

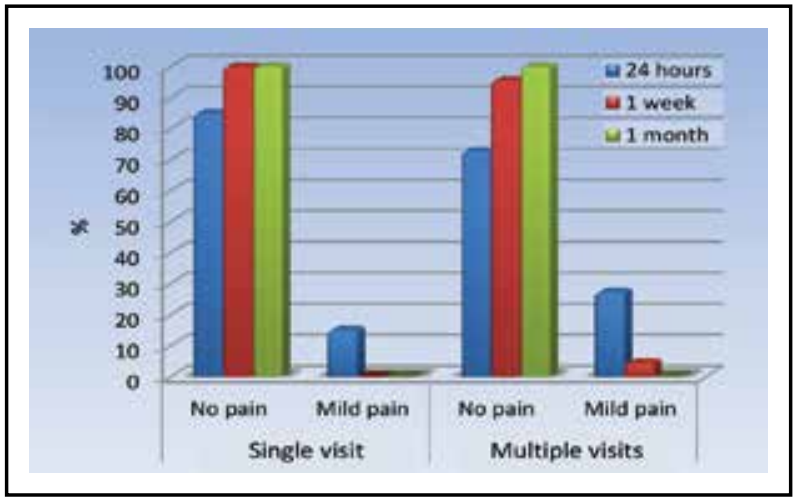

Fig. (1) Histogram showing changes in pain intensity in groups (I\&II).

\section{DISCUSSION}

The major goals of root canal treatment are to clean and shape the root canal system and seal it in three dimensions to prevent reinfection of the tooth. Although initial root canal therapy has been shown to be predictable procedure with a high degree of success, but failures can occur after treatment; $14 \%-16 \%$ failure rates had been reported ${ }^{(20,21)}$.Thus, repeated endodontic treatment requires complex analysis of indications and proper practical execution of the procedure.

Many criteria were selected for current study; one criterion was single rooted teeth with single canal. This type of teeth poses no peculiar difficulty with absence of case-specific hindrance for endodontic retreatment and no morphologic variations that may complicate the retreatment procedures and affect the outcome ${ }^{(22)}$. 
The failed endodontically treated teeth selected for this study was due to inadequate root canal filling procedural error. Inferior technical quality of root canal filling is considered to be the main cause of clinical failure. Other procedural errors were excluded to avoid treatment complication and time consuming for error correction that is not corresponded to treatment protocol ${ }^{(23)}$.

The teeth selected for current study had no acute symptoms (no pain, no swelling, and no pain on percussion) or sinus tract. Teeth that reports preoperative pain is considerably more likely to experience an inter-appointment flare-up than teeth without prior symptoms. The sinus tract may, however, facilitate the influx of bacteria from the oral cavity to colonize the periapex and predispose to extra-radicular infection ${ }^{(24,25)}$.

Successful outcome of the endodontic treatment to be achieved, bacterial populations within the root canal should be ideally eliminated or at least significantly reduced to levels that are compatible with periradicular tissue healing ${ }^{(26)}$. Thus strict aseptic environment was ensured through proper infection control protocol.

Endodontic retreatment aims to remove root canal filling materials from the tooth, revise the shape, and ensure proper cleaning and shaping and obduration of the root canal system. In this study; the old filling was removed using combination of Gates Glidden with Hedstrom files and GP solvent as needed. This technique was reported to be effective in several in vitro and clinical studies ${ }^{(27,28)}$.

There was no statistically significant difference in pain intensity between single and two visits retreatment groups after 24 hours as well as one week ( $\mathrm{P} \leq 0.05)$. Patients experiences less frequency of post-obturation pain after single-visit than those having multiple-visits root canal retreatment. These results corroborated the results of many studies ${ }^{(12-14}$, ${ }^{29)}$.But disagreed with a study reported that the two visits root canal retreatment was more effective in completely eliminating post-operative pain than the single visit technique, however they used variable treatment procedures, and most of cases were treated in two or more appointments ${ }^{(18)}$.

The results in this study refers to decrease in patient numbers who complain of pain with increase of post-obturation time up to one week significantly $(\mathrm{P} \leq 0.05)$, while after one month, the pain completely disappeared in all patient in both groups (I\&II). There was no patient recorded in moderate or severe pain categories at all study periods interval. Thus the post- obturatuion pain was fairly recorded in few cases after one day but that it mostly subsided after one week. This was corroborated with several studies, where they reported that the cases recorded post-operative pain was recorded after 24 hours, the majority of patients in both groups reported no pain or only minimal pain, and pain disappeared after one week of treatment ${ }^{(12-14)}$.

\section{CONCLUSION}

Within the limits of the current study; it can conclude that pain intensity in retreatment patients has reverse proportionally with increase of postobturation time up to one week and completely disappeared after one month. Pain recorded after single and multiple visits retreatment techniques limited to mild pain category.

\section{REFERENCES}

1. Barnes JJ and Patel S. Contemporary endodontics: part 1.Britsh Dent J 2011; 19: 463-8.

2. Lee AHC, Cheung GSP, Wong MCM. Long-term outcome of primary non-surgical root canal treatment. Clinic Oral Invest 2012; 16:1607-17.

3. Siqueira JF, Rôças IN, Ricucci D, Hülsmann M. Causes and management of post-treatment apical periodontitis. $\mathrm{Br}$ Dent J 2014; 216:305-12.

4. Wong AW, Zhang S, Li SK, Zhu X, Chu CH. Incidence of post-obturation pain after single-visit versus multiple-visit nonsurgical endodontic treatment. BMC Oral Health 2015; 15:1-11.

5. Ehrmann EH and Messer H. The relationship of intracanal medicament to post-operative pain in endodontics. Int Endod J 2003; 36:868-75. 
6. Siqueira JF, Rôças IN, Favieri A, Machado AG, Gahyva SM, Oliveira JC. Incidence of Post-operative pain after intracanal procedures based on an antimicrobial strategy. J Endod2002; 28:457-60.

7. UdoyeCh and Aguwa E. Flare-up incidence and related factors in adults. J Dent Oral Hyg 2010; 2: 19-22.

8. Zerella JA, Fouad AF, Spångberg LSW. Effectiveness of a calcium hydroxide and chlorhexidine digluconate mixture as disinfectant during retreatment of failed endodontic cases. Oral Surg Oral Med Oral Pathol Oral Radiol Endod 2005; 100: 756-61.

9. Wong AW, Zhang $\mathrm{C}$, Chu $\mathrm{CH}$. A systematic review of nonsurgical single-visit versus multiple-visit endodontic treatment. Clin Cosmet Invest Dent. 2014; 6:45-56.

10. Soltanoff W.A comparative study of the single-visit and the multiple-visit endodontic procedure. J Endod1978; 4:278-81.

11. Fava LR. One-appointment root canal treatment. Incidence of postoperative pain using a modified double flared technique. Int Endod J 1991; 24:258-62.

12. Jabeen $\mathrm{S}$ and Khurshiduzzaman. A study of post obturation pain following single visit root canal treatment. Chattagram Maa-O- Shishu Hospital Medical College J 2013; 12:16-9.

13. Rao K, Kandaswamy R, Umashetty G, Rathore V, Hotkar C, Patil B. Post-obturation pain following one-visit and two-visit root canal. J Int Oral Health 2014; 6: 28-32.

14. Wong AW, Tsang CS, Zhang S, Li K Y,Zhang C. Treatment outcomes of single-visit versus multiple-visits non-surgical endodontic therapy: a randomized clinical trial. BMC Oral Health 2015; 15: 162 .

15. Oginni A and Udoye CI. Endodontic flare-ups: comparison of incidence between single and multiple visit procedures in patients attending a Nigerian teaching hospital. Odontostomatol Trop2004; 27:23-7.

16. El Mubarak AH, Abu-bakr NH, Ibrahim YE. Post-operative pain in multiple-visit and single-visit root canal treatment. J Endod 2010; 36:36-9.

17. Singh $S$ and AniketGarg A. Incidence of post-operative pain after single visit and multiple visit root canal treatment: A randomized controlled trial J Conserv Dent2012; 15:323-7.

18. Yoldas O, Topuz A, Isci AS, Oztunc H. Postoperative pain after endodontic retreatment: Single-versus two visit treatment. Oral Surg Oral Med Oral Pathol Oral Radiol Endod 2004; 98: 483-7.

19. Price DD, McGrath PA, Buckingham B. The validation of visual analogue scale measures for chronic and exoerimental pain. Pain 1983; 17:45-56.

20. Ng YL, Mann V. Rahbaran S, Lewsey J, Gulabivala K. Outcome of primary root canal treatment: systematic review of literature- part 2: influence of clinical factors. Int Endod J 2007; 41:16-31.

21. Khedmat S. Evaluation of endodontic treatment failure to teeth with periapical radiolucent areas and factors affecting it. J Dent 2004; 1: 34-8.

22. Angerame D, Biasi M, Franco V, Bevilacqua L, Castaldo A. Upper central incisors with periapical lesions treated with two integrated endodontic systems: a six-month randomized controlled trial. J Ital Endod 2016; 30: 33-40.

23. Ng YL, Mann V, Gulabivala K. A prospective study of the factors affecting outcome of nonsurgical root canal treatment: part 1; Periapical health. Int Endod J 2011; 44: 583-609.

24. Walton R E. Inter-appointment flare-ups: incidence, related factors, prevention, and management. Endod Topics 2002; 3: $67-76$

25. Moazami F, Sahebi S, Sobhanamayan F, Alipour A. Success rate of nonsurgical endodontic treatment of nonvital teeth with variable periradicular lesions. Iranian Endod J 2011; 6: 119-24.

26. Siqueira JF and Rôças IN. Clinical implications and microbiology of bacterial persistence after treatment procedures. J Endod 2008; 34: 1201-1301.

27. Zuolo AS, Mello Jr JE, Cunha RS, Zuolo ML, Bueno CE. Efficacy of reciprocating and rotary techniques for removing filling material during root canal retreatment. Int Endod J 2013; 46: 947-53.

28. Ali SG, Mulay S, Palekar A, Sejpal D, Joshi A, Gufran $\mathrm{H}$. Prevalence of and factors affecting post-obturation pain following single visit root canal treatment in Indian population: A prospective, randomized clinical trial. Contemp Clin Dent.2013; 3: 459-63.

29. Gill GS, Bhuyan AC, Bhuyan D. Single versus multi-visit endodontic treatment of teeth with apical periodontitis: An in vivo study with 1 year evaluation. Ann Med health Sci Res 2016; 6: 19-26. 